\title{
Oscillatory rheology of dense, athermal suspensions of nearly hard spheres below the jamming point
}

\author{
Christopher Ness, ${ }^{1}$ Zhongyang Xing, ${ }^{2}$ and Erika Eiser ${ }^{2}$ \\ ${ }^{1}$ Department of Chemical Engineering and Biotechnology, \\ University of Cambridge, Cambridge CB3 OAS, United Kingdom \\ ${ }^{2}$ Cavendish Laboratory, University of Cambridge, Cambridge CB3 OHE, United Kingdom
}

(Dated: April 13, 2017)

\begin{abstract}
The viscosity of a dense suspension has contributions from hydrodynamics and particle interactions, both of which depend upon the flow-induced arrangement of particles into fragile structures. Here, we study the response of nearly hard sphere suspensions to oscillatory shear using simulations and experiments in the athermal, non-inertial limit. Three distinct regimes are observed as a function of the strain amplitude $\gamma_{0}$. For $\gamma_{0}<10^{-1}$, initially non-contacting particles remain separated and the suspension behaves similarly to a Newtonian fluid, with the shear stress proportional to the strain rate, and the normal stresses close to zero. For $\gamma_{0}>10^{1}$, the microstructure becomes well-established at the beginning of each shear cycle and the rheology is quasi-Newtonian: the shear stress varies with the rate, but flow-induced structures lead to non-zero normal stresses. At intermediate $\gamma_{0}$, particle-particle contacts break and reform across entire oscillatory cycles, and we probe a non-linear regime that reveals the fragility of the material. Guided by these features, we further show that oscillatory shear may serve as a diagnostic tool to isolate specific stress contributions in dense suspensions, and more generally in those materials whose rheology has contributions with both hydrodynamic and non-hydrodynamic origin.
\end{abstract}

\section{INTRODUCTION}

Flowing amorphous materials such as dense suspensions and pastes find applications across industry, yet a complete microscopic description of their mechanical behaviour remains the subject of much study and debate [1]. Though seemingly distinct at a phenomenological level, many types of structurally disordered materials share underlying physical features, and their rheology is increasingly unified by their positioning within the jamming phase diagram [2-4]. Those systems below jamming are further unified according to simple phase diagrams that take into account particle repulsion and attraction, inertia, and friction [5-8].

Such systems often also share the common feature of fragility [9]: loads that are compatible with the microstructural configuration can be sustained; incompatible loads, however temporary, lead to rapid particle reorganisation. Materials in fragile states can be forced to unjam or rearrange and flow by small changes in shearing direction. The concept of fragility is also useful for explaining the flowing behaviour of dense suspensions, which, while not jammed themselves, may comprise fragile force chains that can engage under compatible loads or collapse under incompatible ones giving the material a highly anisotropic viscosity $[10,11]$. While this detail has been exploited recently to tune the flow response of suspensions [12], fluctuations or intermittent shearing can consequently coincide with highly non-linear rheology, relevant to flow instabilities such as shark toothing during paste extrusion, for example [13]. To this end, oscillatory rheology, which has classically been employed to study viscous and elastic contributions to rheology across a broad class of amorphous material [14-16], might prove useful in suspension rheology not only to examine the material response to non-steady flows in practice [17], but also to reveal the inherent fragility and hence provide information linking microstructure to bulk rheology. Though the presence of fragility has been brought into question for jammed states of softer particles [18, 19], it remains an instructive concept for understanding the roles of microstructure and contact and hydrodynamic stresses in disordered materials below jamming [20].

Dimensional analysis suggests that the rheology of athermal, non-inertial, hard-sphere suspensions is rateindependent [21-23] in the limit of large strains. The flow remains non-Newtonian, though, in that arising nonzero normal stresses are linked to the solids volume fraction $\phi$ through the viscous number $I_{v}$ [24], an analogue of the inertial number $I[25,26]$. Experimental evidence suggests, however, that even within this supposedly rateindependent limit, dense suspensions may have stress contributions arising from particle-particle interactions as well as hydrodynamics [20, 27]. Rate-independence holds for stresses that are not close to $\sigma^{*}$, a threshold stress for the onset of friction in such particle-particle surface contacts, which enhances flow resistance [6]. We expect, therefore, that the contact contribution to the stress increases near $\sigma^{*}$ (manifested as shear thickening [28]), but that rate-independence holds elsewhere.

Below the jamming point, a finite strain is required for assembly of load bearing contact structures [10], illustrated in Figure 1. At smaller strains, the microstructural state might represent some intermediate between successive load bearing states, that is itself unable to sustain large stresses. Here, hydrodynamic stresses may dominate, leading to apparently viscous rheology. Ramping up the strain, we see an onset of load bearing contacts at intermediate values (hitherto referred to as strain stiffening [29]), and the above mentioned rate-independent, 
quasi-Newtonian regime for large strains. This sequence of microstructural events may be usefully interpreted as a nonlinear response to oscillatory flow, as previously demonstrated in the context of soft colloidal glasses [30]. Oscillatory measurements in suspensions might, therefore, reveal viscous rheology at very small or large strains, with nonlinearities arising for intermediate values.

Due to disordered crowding at the microscale, the particles (e.g. the suspended solids in a suspension) do not follow an affine trajectory as defined by the bulk material deformation, as would be the case for a lattice solid [31]. Instead, particle-particle interactions, which do not necessarily obey any kind of spatial or temporal symmetry, necessitate nonaffine trajectories at the particle level at sufficiently large strains [32,33]. For $\phi<\phi_{c}$ the particleparticle interactions that give rise to nonaffinity are further responsible for disrupting the time-symmetry of the flow [34], as was beautifully demonstrated again in a recent revisiting of Taylor's classical experiment [35]. In addition to a bulk rheological nonlinearity, this affine-tononaffine transition in particle trajectories above a critical strain might serve as a microstructural signature of the intermediate strain amplitude regime.

In this article, we set out the response of nearly hard sphere suspensions to oscillatory shear, taking evidence from simulations and experiments to demonstrate the behaviour under small-, medium- and large-amplitude strains. As discussed above, and in accordance with a series of works $[10,11,20]$, it has proven highly instructive to consider the response of suspensions to transient flows as a strain-dependent series of microstructural events. Within this framework, the interpretation of large-amplitude oscillatory shear (LAOS) rheology data is challenging and as such there is an ongoing discussion in the literature on how one can understand the nonlinearities associated with LAOS data [36]. Initially, data were analysed in terms of an oscillatory time series, with stress responses being interpreted as superpositions of periodic contributions with varying phase shifts that can be extracted through Fourier transformations [37]. Later attempts to add a physical meaning to such superpositions included the replacement of sinusoidal contributions with exotic characteristic basis functions such as rectangular and triangular waves [38], the stress decompostion approach [39], leading to the models of Ewoldt and McKinley [40] that offer an interpretation of elastic and viscous stresses as sets of orthogonal polynomials. More recently, the 'sequence of physical processes' framework [41] has proven a successful approach to capture the nonlinear response of yield-stress and visco-elastic materials within the strain, strain rate and stress domain. Given the lack of clear consensus in the literature regarding methods of analyzing oscillatory rheology data for suspensions, however, we refrain from making a specific choice from the above models, and instead focus on the overall transient shear stress response and how structural rearrangements give rise to the rheological forms observed.

We find Newtonian behaviour for small strains, where particles remain separated by viscous lubrication films. In terms of a generalised non-Newtonian number proposed by Giacomin [42], we expect that this regime corresponds to a number close to zero, since nonlinearities associated with either amplitude or frequency effects are absent. For large strains the response is quasiNewtonian: the Lissajous curve shows superficially viscous behaviour, but the suspension is within the abovementioned rate-independent regime, where both hydrodynamic and contact stresses contribute to the overall rheology. At intermediate strains, building and destroying particle contacts [9] leads to highly non-linear behaviour in all stresses and microstructural quantities. We finally demonstrate that oscillatory rheology can complement shear reversal protocols $[10,11,20,43]$ to diagnose shear-induced structural contributions to the viscosity of dense suspensions. This will be useful for characterising suspended particles in material products as diverse as industrial ceramic pastes and catalytic washcoats.

\section{METHODOLOGY}

\section{A. Numerical simulation}

Force and stress calculation We consider dense suspensions with solid volume fractions ranging from $\phi=$ $0.50 \rightarrow 0.62$. The material is represented by $N_{p}=$ $\mathrm{O}\left(10^{3}\right)$ bidisperse, spherical particles (diameters $d$ and $1.4 d$, density $\rho$ ) in a cubic periodic domain of volume $V$. Newtonian dynamics are calculated in a stepwise, deterministic manner using an established molecular dynamics code [44], applying a normal penalty function $\mathbf{F}_{\alpha}^{c, n}=k \varrho \mathbf{r}_{\alpha} /\left|\mathbf{r}_{\alpha}\right|$ with stiffness $k$ to minimize overlaps (with magnitude $\varrho$ ) between contacting particle pairs that have centre-to-centre vectors $\mathbf{r}_{\alpha}$. We further add a resistance to pairwise tangential motion $\mathbf{F}_{\alpha}^{c, t}=k \mathbf{u}_{\alpha}$, for tangential displacement $\mathbf{u}_{\alpha}$, constrained by a coefficient of friction $\mu$ such that $\left|\mathbf{F}_{\alpha}^{c, t}\right| \leq \mu\left|\mathbf{F}_{\alpha}^{c, n}\right|$ [45]. The particles are suspended in a density-matching liquid, accounted for by resolving frame-invariant hydrodynamic lubrication forces $\mathbf{F}_{\beta}^{h}$ with leading term $-a_{s q} 6 \pi \eta_{f} \mathbf{v}_{\beta}$, for neighbouring particle pairs (within a cutoff length $0.05 d$, the sensitivity to which we tested earlier [7]) with centreto-centre vector $\mathbf{r}_{\beta}$, relative normal velocity $\mathbf{v}_{\beta}$, fluid viscosity $\eta_{f}$ and squeeze resistance $a_{s q}$. A detailed description of the simulation model is given elsewhere [46]. The bulk contact and hydrodynamic stress tensors are calculated according to

$$
\boldsymbol{\sigma}^{c}=-\frac{1}{V} \sum_{\alpha=1}^{N_{c}} \mathbf{r}_{\alpha}\left(\mathbf{F}_{\alpha}^{c, n}+\mathbf{F}_{\alpha}^{c, t}\right),
$$

and

$$
\boldsymbol{\sigma}^{h}=-\frac{1}{V} \sum_{\beta=1}^{N_{h}} \mathbf{r}_{\beta} \mathbf{F}_{\beta}^{h}
$$


respectively, where $N_{c}$ and $N_{h}$ are the number of contacting and hydrodynamically interacting pairs, respectively. The total stress is given by $\boldsymbol{\sigma}=\boldsymbol{\sigma}^{c}+\boldsymbol{\sigma}^{h}$. Data are presented for the shear $\left(\sigma_{x y}\right)$ and normal $\left(\sigma_{x x}, \sigma_{y y}, \sigma_{z z}\right)$ components of $\boldsymbol{\sigma}$ as well as $\sigma_{x y}^{c}$ and $\sigma_{x y}^{h}$.

Sample preparation and oscillatory shearing Samples are prepared using an energy minimisation scheme comprising thermal equilibration, leading to spatially isotropic suspensions with no initial particle-particle contacts. The simulation box is subjected to an oscillatory shear deformation of amplitude $\gamma_{0}$, with flow and gradient in $x$ and $y$ respectively (see Figure 1). The time-dependent strain and rate of strain are given by $\gamma(t)=\gamma_{0} \sin (\omega t)$ and $\dot{\gamma}(t)=\gamma_{0} \omega \cos (\omega t)$, respectively. The strain amplitude $\gamma_{0}$ is varied between $10^{-3}$ and $10^{1}$ to explore the linear and non-linear rheological responses of the suspension. Although, as discussed in the Introduction, we interpret the material response throughout as series of microstructural events, as opposed to superpositions of multiple bulk periodic functions, we find it instructive to model the resulting shear stress according to $\sigma_{x y}(t)=\sigma \sin (\omega t+\delta)$, which gives representative storage $\left(G^{\prime}\right)$ and loss $\left(G^{\prime \prime}\right)$ moduli for the suspension as

$$
G^{\prime}=\frac{\sigma}{\gamma_{0}} \cos \delta \quad(3) \quad G^{\prime \prime}=\frac{\sigma}{\gamma_{0}} \sin \delta
$$

from which we may recover an approximation of the complex viscosity magnitude according to

$$
\left|\eta^{*}\right|=\left(G_{1}^{\prime 2}+G_{1}^{\prime \prime 2}\right)^{1 / 2} / \omega .
$$

We determined delta simply by using a best-fitting algorithm to approximate the non-sinusoidal material response as a sinusoidal one. This is a very good approximation in the small- and large-amplitude limits where the response is close to linear, but it necessarily averages over the nonlinearities for intermediate amplitudes. Moreover, we find the complex viscosity magnitude itself (without explicitly considering the phase) a useful parameter to consider alongside the microstructural evolution. Starting from the isotropic equilibrated assembly, the samples are sheared for ten oscillatory cycles. The following results represent ensemble averages across 40 realisations, for the final five cycles. Notably, we did not observe any long term effects when shearing the sample for additional cycles. In fact, the long-time response is obtained within the first half of the first cycle in all cases. This is in contrast to a recent study of dry grains under oscillatory flow, in which rich phase behaviour was observed after many shear cycles [47]. We attribute this disparity to our use of bidisperse grains, which frustrate ordering, and fixed volume, which prevents contraction and dilation.

The dimensional parameters in the model and their units are particle density $\rho\left[\right.$ mass $/$ length $\left.^{3}\right]$, particle diameter $d$ [length], fluid viscosity $\eta_{f}$ [mass/(length $\times$ time $\left.)\right]$, particle stiffness $k$ [mass/time $\left.{ }^{2}\right]$ and shear rate $\dot{\gamma}(t)$ [1/time] (which is related to frequency $\omega[\mathrm{rad} /$ time $]$ ), from which we construct two dimensionless control parameters, $\rho \dot{\gamma}(t) d^{2} / \eta_{f}$ and $\dot{\gamma}(t) d / \sqrt{k / \rho d}$. We choose $\omega$ such that both parameters remain $\ll 1$ for all $t$, giving non-inertial and nearly hard sphere rheology, respectively. The suspension viscosity and microstructural evolution under well-established flow are therefore expected to be rate-independent [21]. Shear stresses that resist flow are positive for positive $\dot{\gamma}$, while compressive normal stresses are negative independent of the sign of $\dot{\gamma}$.

\section{B. Experimental protocol}

Sample preparation Experimental results presented here are obtained using supermarket-bought cornstarch particles supended at $50 \mathrm{wt} . \%$ in a mixture that is itself composed of 50 wt. $\%$ water and 50 wt. $\%$ glycerol (suspending liquid viscosity $\eta_{f}=0.012 \mathrm{~Pa} . \mathrm{s}$, density $\rho=$ $\left.1.1 \mathrm{~g} / \mathrm{cm}^{3}\right)$. The steady shear rheology of our suspension is quantitatively consistent with recent works in shear thickening of cornstarch suspensions [48, 49]. Given the acute size dependence demonstrated recently [6], therefore, we expect that such agreement is indicative of comparable particle sizes and assume a particle diameter of order $10 \mu \mathrm{m}$. The samples were freshly prepared before each experiment and thoroughly dispersed with a vortex mixer then rested for several minutes before loading.

Oscillatory shearing protocol Rheological measurements were performed using a stress-controlled rheometer (Anton Paar MCR501) in a cone-plate geometry with diameter $50 \mathrm{~mm}$ and angle $2^{\circ}$ at a temperature of $12^{\circ} \mathrm{C}$ [50]. The temperature control was achieved by a Peltier system consisting of the bottom plate and a hood with Peltier elements equipped on each. Oscillatory strain sweep measurements were performed with strain amplitudes $\gamma_{0}=0.1 \rightarrow 5$, under a fixed angular frequency of $\omega=50 \mathrm{rad} / \mathrm{s}$, ensuring the characteristic stress $\sigma$ exceeded the onset for frictional particle contacts $\sigma^{*}$. Frequency sweeps ranging from $\omega=0.5 \rightarrow 50 \mathrm{rad} / \mathrm{s}$ were performed with strain amplitudes $\gamma_{0}=0.5$ and $\gamma_{0}=2$. Measurements were taken across a minimum of 100 oscillatory cycles in each case. The Péclet number remains at all times $\gg 100$ such that Brownian motion can be neglected. The influence of sample sedimentation and evaporation is non-negligible after $\approx 60$ mins, therefore all the presented data were taken within this time duration. No evidence of particle aggregation was observed during the experiment. It is noted that shear history effects may arise in the experiment that are not accounted for in simulation, though we verified that quantitatively consistent experimental results were obtained for both upwards and downwards sweeps of amplitude and frequency. 


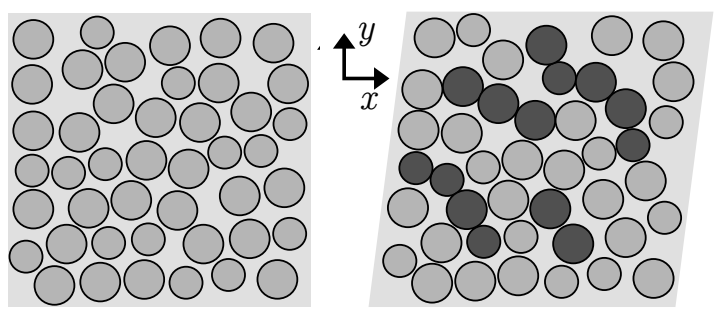

FIG. 1. Schematic of particle assembly in the unsheared state (left) and in the sheared state (right). Dark shading indicates force-transmitting particle contacts that appear after some necessary strain. Shown in 2-dimensions for clarity; all simulation data are obtained from 3-dimensional suspensions. Inset: coordinate definition used throughout.

\section{RESULTS}

In Figure 2 we present computational and experimental results of the bulk rheological response of the suspension to oscillatory shear, for increasing $\gamma_{0}$ at fixed $\omega$. Shown are computational results for frictionless $(\mu=0)$ and frictional $(\mu=1)$ particles, both at $\phi=0.55$, and experimental results measured at $\omega=50 \mathrm{rad} / \mathrm{s}$ for which the characteristic shear stress $\sigma$ exceeds the onset stress for frictional contacts $\sigma^{*}[6,49]$. The numerically predicted storage and loss moduli, particularly for frictional particles, show remarkable qualitative agreement with experiment. Indeed, the limiting complex viscosity magnitudes are in good quantitative agreement, though there is some degree of offset in the critical strain magnitude for the viscosity increase, Figure 2d. Such an offset might be explained by the disparity in precise volume fractions, which is challenging to overcome for cornstarch suspensions [29].

The detailed shear stress response, predicted by simulation at $\mu=0$ and $\mu=1$, is given in the form of Lissajous curves in Figure 3 at $\phi=0.55$ for five representative values of $\gamma_{0}$. Shown in Figure 4 for $\mu=0$ are the evolutions of per-particle contact number $Z_{c}=2 N_{c} / N_{p}$ and two quantifications of the suspension fabric, defined according to directly contacting particles and hydrodynamically interacting particles, given respectively as

$$
\boldsymbol{A}^{c}=-\frac{1}{N_{c}} \sum_{\alpha=1}^{N_{c}} \mathbf{r}_{\alpha} \mathbf{r}_{\alpha} \quad(6) \quad \boldsymbol{A}^{h}=-\frac{1}{N_{h}} \sum_{\beta=1}^{N_{h}} \mathbf{r}_{\beta} \mathbf{r}_{\beta}
$$

from which we report the $x y$ components, written as $A^{c}$ and $A^{h}$ for convenience. Contacts aligned in the compressive quadrant have positive fabric contributions for positive shear rates, Figure 1b. In Figure 5 we illustrate the reversibility and affinity of the particle displacements with increasing $\gamma_{0}$. Shown in Figure 6 are the normal stresses and normal stress differences $N_{1}=\sigma_{x x}-\sigma_{y y}$ and $N_{2}=\sigma_{y y}-\sigma_{z z}$ corresponding to $\mu=0$ in Figure 3. Note that in each case the simulation results are rate-independent. In Figure 7 we present experimental results for frequency sweeps at $\gamma_{0}=0.5$ and $\gamma_{0}=2$, while in Figure 8 we propose representative Pipkin diagrams at two volume fractions.

\section{A. Newtonian response at small amplitude}

In the limit of small $\gamma_{0}$, the simulation predicts purely viscous rheology, with $\sigma_{x y} \propto \dot{\gamma}$, indicated by a circle in the stress-strain Lissajous plot, Figure 3 , and $G_{1}^{\prime \prime} \gg G_{1}^{\prime}$, Figure 2. This is in good agreement with the experimental result. The contact contribution to the stress is observed to be negligible. Particles remain separated by lubrication films that dominate the dissipation and merely oscillate about their initial positions with no plastic rearrangement. This is illustated by $Z_{c} \approx 0$, Figure 4 , and in Figure 5a, which gives the net displacement of a particle with position after one cycle $(\omega t=2 \pi) \mathbf{x}_{2 \pi}$ from its starting position $\mathbf{x}_{0}$, where $\langle\ldots\rangle$ denotes the average over all particles and realisations. In the limit of small $\gamma_{0}$, particle displacements are demonstrated to be reversible. Similarly, for small $\gamma_{0}$, the absence of direct particle contacts means individual particle motions are not highly constrained, so their translation under oscillatory flow tends to follow the affine deformation of the simulation box, so fluctuating, or non-affine, velocities are small. In Figure 5b we plot the particle-averaged nonaffine velocity magnitudes at the end of the shearing cycle, defined for particle $i$ with position $\mathbf{x}_{i}$ and velocity $\mathbf{v}_{i}$ as $\mathbf{v}^{\prime}=\mathbf{v}_{i}-\dot{\gamma} \mathbf{x}_{i}$. While $Z_{c}$ remains very close to 0 , $A^{c}$ is undefined during most of the cycle. The overall structure remains largely isotropic, with $A^{h} \rightarrow 0$. Normal stresses remain close to zero for small $\gamma_{0}$, so that the rheological response might be described as Newtonian. This is in contrast to the large $\gamma_{0}$ limit, described later, that we refer to as quasi-Newtonian.

The small strain limit holds while the particle translations induced by the shear flow remain smaller than the mean particle separations developed during sample preparation. Indeed, the transition to the irreversible region occurs for smaller $\gamma_{0}$ with increasing $\phi$, as expected (Figure 5). In this small strain regime, therefore, there is no frequency at which particle contacts will arise, so the relaxation time is always vanishing. As a result the viscous response retains rate-independence provided we keep $\rho \dot{\gamma}(t) d^{2} / \eta_{f}, \dot{\gamma}(t) d / \sqrt{k / \rho d}<1$.

\section{B. Non-linear response at moderate amplitude}

The stress response becomes non-linear when the strain magnitude approaches then exceeds $10^{-1}$, evidenced by a negative surge in the lower right quadrant of the Lissajous curves at $\gamma_{0}=0.22$ and $\gamma_{0}=0.55$, Figure 3a. Here, particle contacts begin to become widespread, with particles contacting at least one of their neighbours on average at the peak strain, Figure 4a. Over these strain amplitudes, such particle-particle interac- 

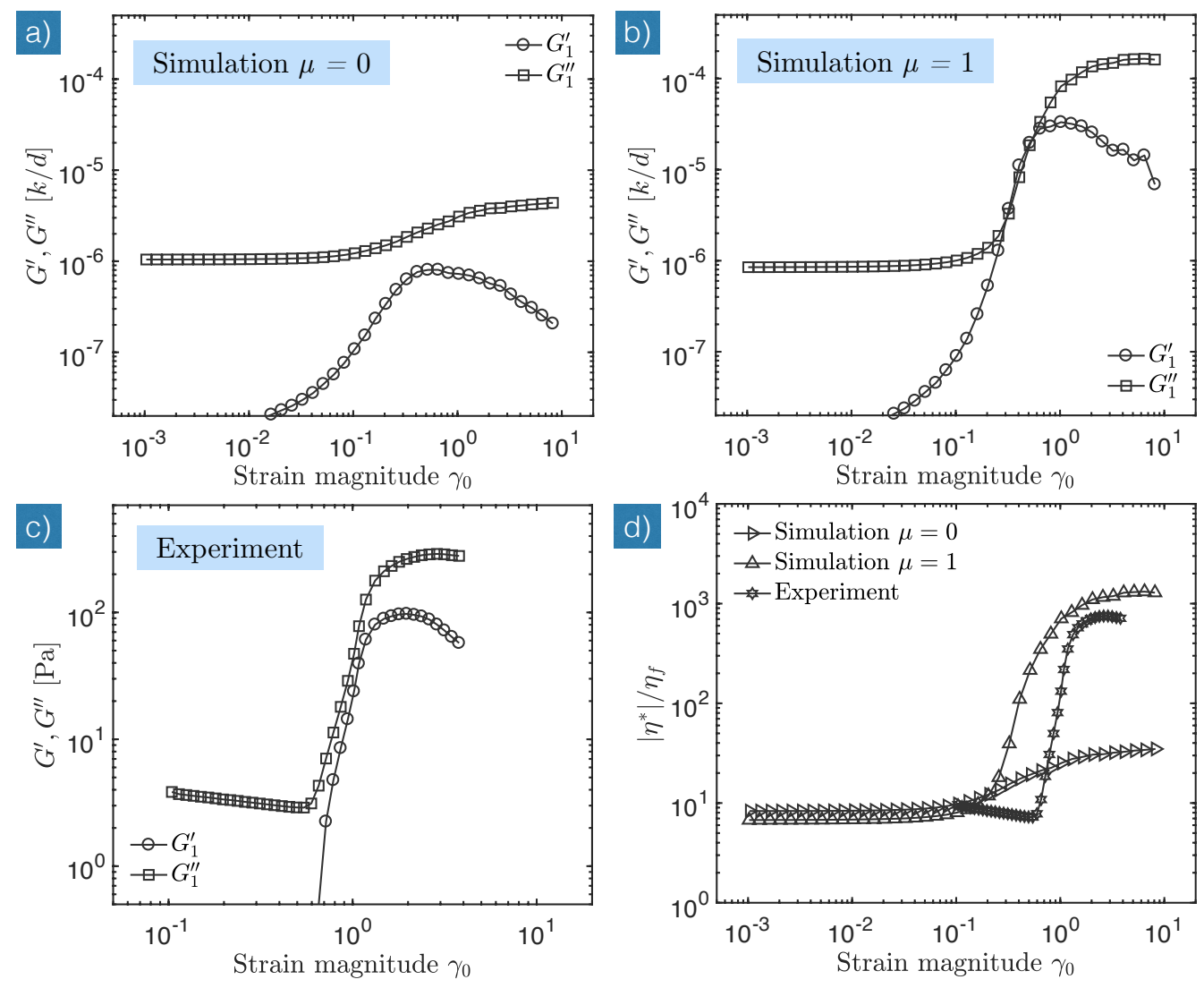

FIG. 2. Storage $\left(G^{\prime}\right)$ and loss $\left(G^{\prime \prime}\right)$ moduli and complex viscosity magnitude $\left(\left|\eta^{*}\right|\right)$ as functions of $\gamma_{0}$ for a dense suspension of nearly hard particles. (a) Simulated suspension with friction coefficient $\mu=0$, volume fraction $\phi=0.55$; (b) Simulated suspension with friction coefficient $\mu=1$, volume fraction $\phi=0.55$; (c) Cornstarch suspension at $\sigma>\sigma^{*}$ where we expect frictional contacts; (d) Complex viscosity magnitude for each suspension. Shown are the storage and loss moduli, defined according to Equations 3-4 and the complex viscosity magnitude calculated according to Equation 5. The presented moduli are given in units of $k / d$ and $\mathrm{Pa}$, while the viscosity is scaled by the suspending fluid viscosity $\eta_{f}$.

tions begin to generate contact stresses that contribute to the total stress out-of-phase with the hydrodynamic stresses, which manifests as $G_{1}^{\prime}$ approaching $G_{1}^{\prime \prime}$ and a gradual increase of the suspension viscosity magnitude $\left|\eta^{*}\right|$, Figure 2. The larger deformations now lead to large scale organisation of the microstructure, illustrated by the emergent switching of $A^{h}$, and the loss of reversibility in particle trajectories, Figure 5a.

The reconfiguration of the particle contact network on this scale further requires particle motions that deviate from the net shearing flow. Moreover, direct particleparticle contacts lead to unbalanced, anisotropic forces on particles that must be dissipated by nonaffine motions, contributing to an increased suspension viscosity [51]. Linked to this, there is a sudden increase in the magnitude of the non-affine velocities $\mathbf{v}^{\prime}$ when $\gamma_{0}>10^{-1}$. The nonaffinity increases with $\phi$ as jamming is approached, as particles must deviate further from their affine trajectories to satisfy the imposed shear rate. This result is reminiscient of several other findings and theoretical models $[4,51]$. Though in general for disordered systems the emergence of nonaffinity and irreversibility are not neces- sariliy equivalent, our results indicate that the onsets of both are correlated in this case. Precisely understanding the link (or lack thereof) between these two phenomena for below-jamming systems could be a promising line of future investigation and could indeed serve to enhance the unification of disordered systems across the jamming transition.

A sudden loss of contacts coupled with a discontinuity in $A^{c}$ is observed at $\dot{\gamma}=0$, corresponding to the turnaround of the flow direction in the suspension. The distorted microstructure, represented by both $A^{h}$ and $A^{c}$, that appears under moderate strain amplitudes becomes immediately incompatible with a reversed shearing direction, allowing a total relaxation of direct particle-particle contacts, resulting in loss of the contact stress contribution and an instantaneous viscous flow with greatly reduced stress. The structural information stored in $A^{c}$ is lost at this point, since it is undefined when $N_{c} \rightarrow 0$. By contrast, the hydrodynamic fabric $A^{h}$, which represents the mean orientation of neighbouring particle pairs, remains even when the contacts themselves vanish. We observe some continuity, therefore, in the evolution of the 
a) $\mu=0$
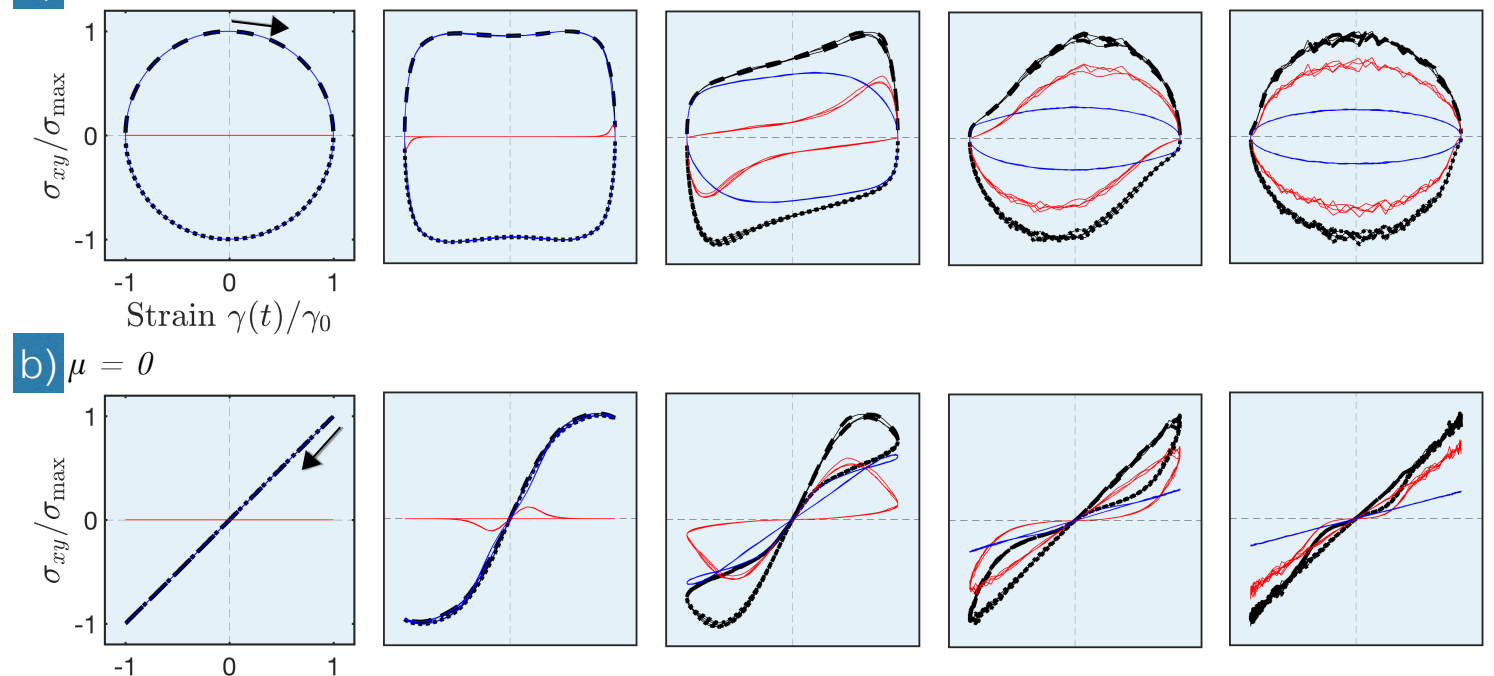

Strain rate $\dot{\gamma}(t) / \gamma_{0} \omega$

\section{c) $\mu=1$}
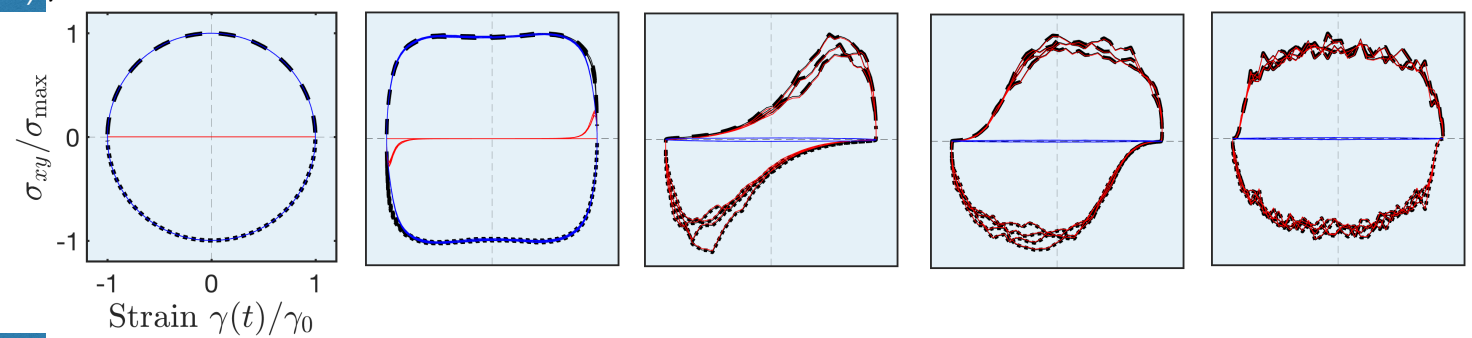

d) $\mu=1$
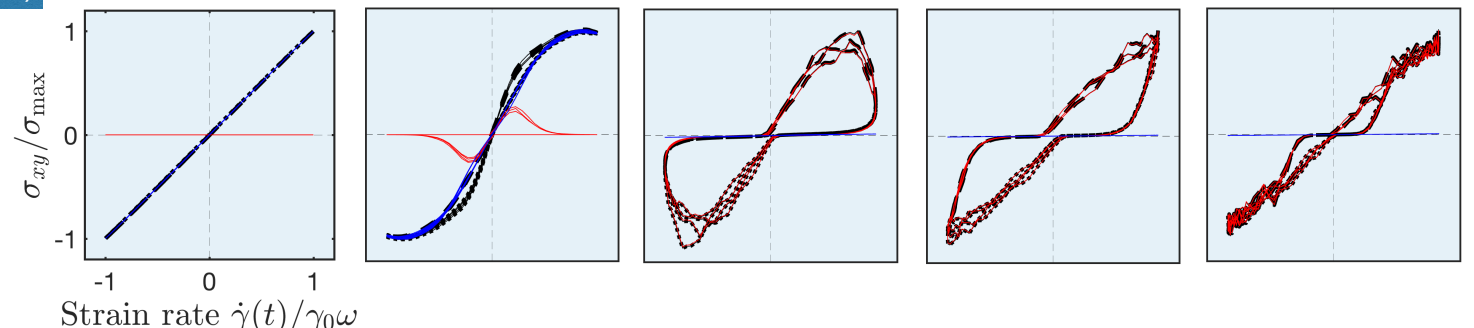

Strain rate $\dot{\gamma}(t) / \gamma_{0} \omega$

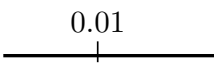

0.22

0.55

1.39

8.79

Increasing strain amplitude $\gamma_{0}$

FIG. 3. Rheological data for oscillatory shear in a dense suspension, predicted by simulation for $\mu=0$ and $\mu=1$ at $\phi=0.55$. Black lines indicate total shear stress; blue lines indicate hydrodynamic contribution; red lines indicate contact contribution. (a) Stress-strain relationship for $\mu=0$; (b) Stress-strain rate relationship for $\mu=0$; (c) Stress-strain relationship for $\mu=1$; (d) Stress-strain rate relationship for $\mu=1$; Shown are data for increasing $\gamma_{0}$, left to right, with the axis labels in the far left applicable to their whole row. To indicate the phase of each set of data, results corresponding to $\dot{\gamma}(t)>0$ are highlighted with dashes, while those for $\dot{\gamma}(t)<0$ are dotted. Arrowheads in (a) and (b) indicate starting point and direction to read, for initial increase of the strain. Strain amplitudes are indicated along the bottom axis.

microstructure at this scale during flow reversal.

The onset of widespread particle contacts corresponds to the emergence of significant normal stresses, Figure 6. Consistent with previous findings [11], we observe that the deformation of the contact network for increasing $\gamma(t)$ requires squeezing of lubrication films, generating compressive normal stresses and positive $N_{1}$, indicative of dilatant behaviour. This is best illustrated for the $\gamma_{0}=0.22$ case, where we observe positive $N_{1}$ (and negative $N_{2}$ ) for increasingly negative $A^{h}$. Upon reversal of the flow direction, there is a very slightly negative $N_{1}$ and a larger positive $N_{2}$, both indicating slight tensile behaviour, as the large scale microstructural orientation returns to isotropic, illustrated by $A^{h}$ returning to zero. 

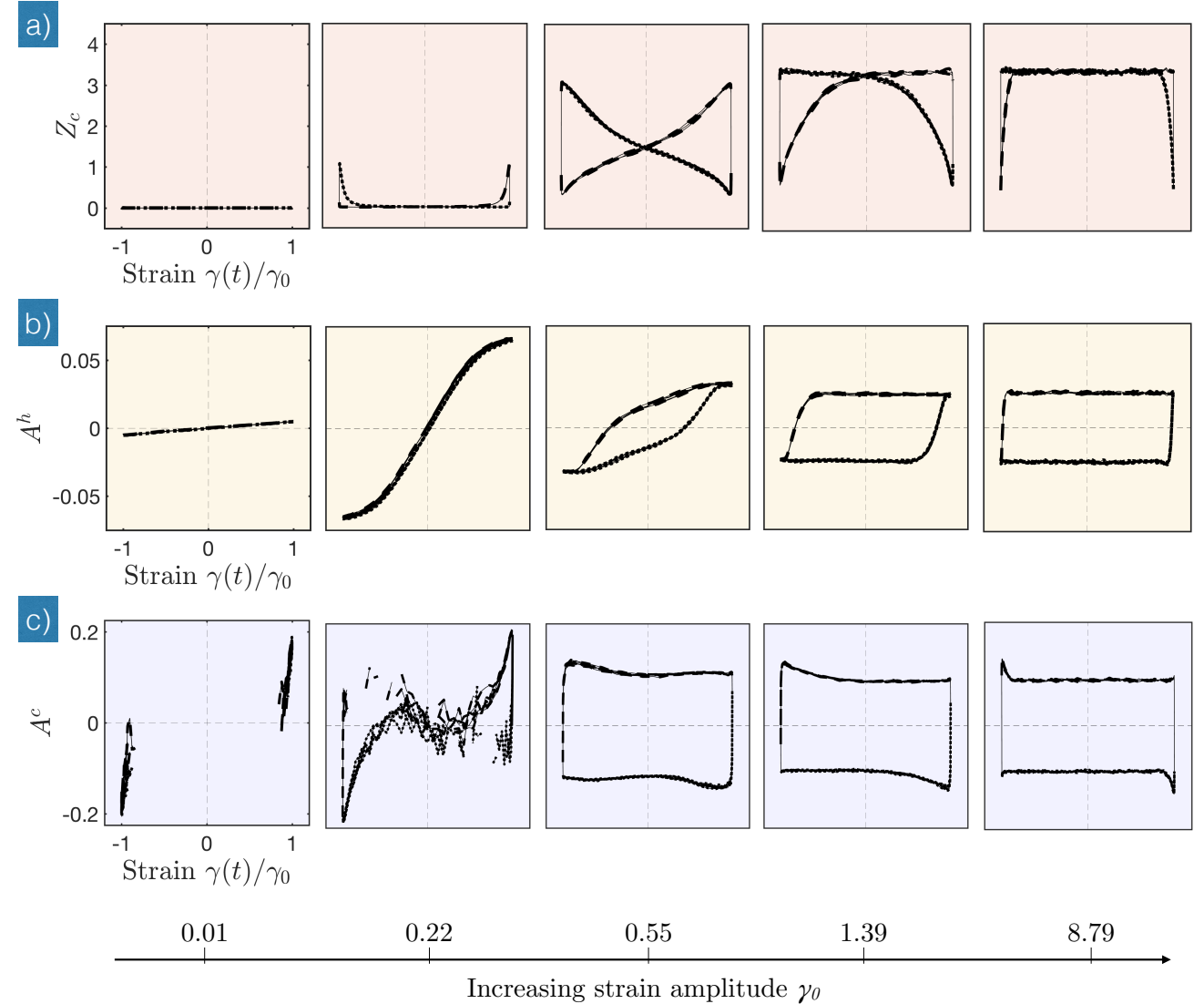

FIG. 4. Microstructural data for oscillatory shear in a dense suspension, predicted by simulation for $\mu=0$ and $\phi=0.55$. (a) Evolution of coordination number $Z_{c}$; (b) Hydrodynamic fabric $A^{h}$; (c) Contact fabric $A^{c}$. Shown are data for increasing $\gamma_{0}$, left to right, with the axis labels in the far left applicable to their whole row. To indicate the phase of each set of data, results corresponding to $\dot{\gamma}(t)>0$ are highlighted with dashes, while those for $\dot{\gamma}(t)<0$ are dotted.
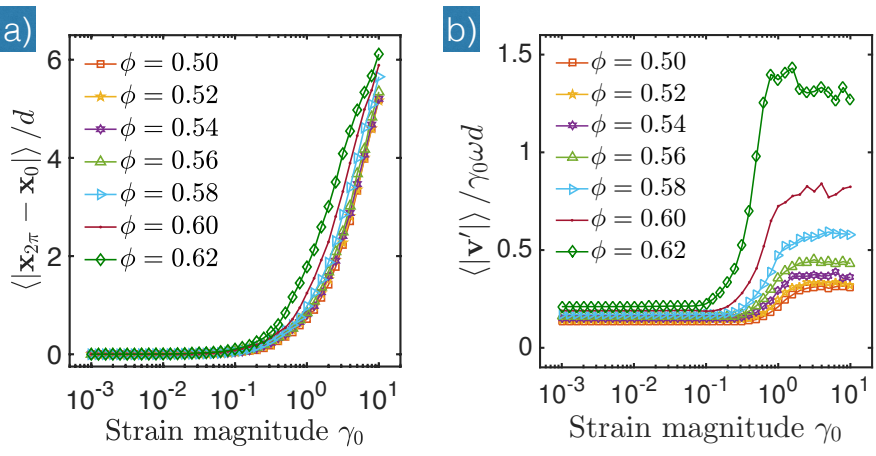

FIG. 5. Irreversible and non-affine particle translation with increasing $\gamma_{0}$ during oscillatory shear as predicted by simulation for $\mu=0$ and $\phi=0.55$. (a) Particle-averaged net translation after one cycle of shearing. Particles return exactly to their starting positions for $\gamma_{0}$ up to 0.1. For larger $\gamma_{0}$, plastic rearrangements lead to a loss of reversibility; (b) Particle-averaged non-affine velocity magnitude (rescaled by a characteristic streaming rate $\gamma_{0} \omega d$ ) after one cycle of shearing. Spatially anisotropic particle-particle contacts at large $\gamma_{0}$ lead to unbalanced forces on particles, that dissipate by non-affine motions. We find equivalent results for $\mu=1$.
For strain magnitudes in the range $10^{-1}<\gamma_{0} \lesssim 10^{0}$, it is noted that the stresses and microstructural quantities retain strain dependent characteristics at all stages of the oscillatory cycle, indicating that the flow never achieves a well-established state, but rather remains in a perpetual transient configuration. In this respect, we may state that full coherence between the stress/strain state and the microstructural details is only achieved towards the end of each oscillatory cycle (if at all), meaning the suspension is fragile with respect deformations in any direction. Although the complete change in flow direction adopted here is at odds with the very small change in shear direction required to indicate the presence of fragility, we find that the rapid changes in stress response for very small amounts of strain do indeed render this material fragile. The response to moderate strain amplitudes is in contrast to the case where $\gamma_{0} \gtrsim 10^{1}$, discussed below, where the suspension achieves a wellestablished state, i.e. the extent and arrangement of the contact network reaches steady state near the start of each oscillatory cycle. 

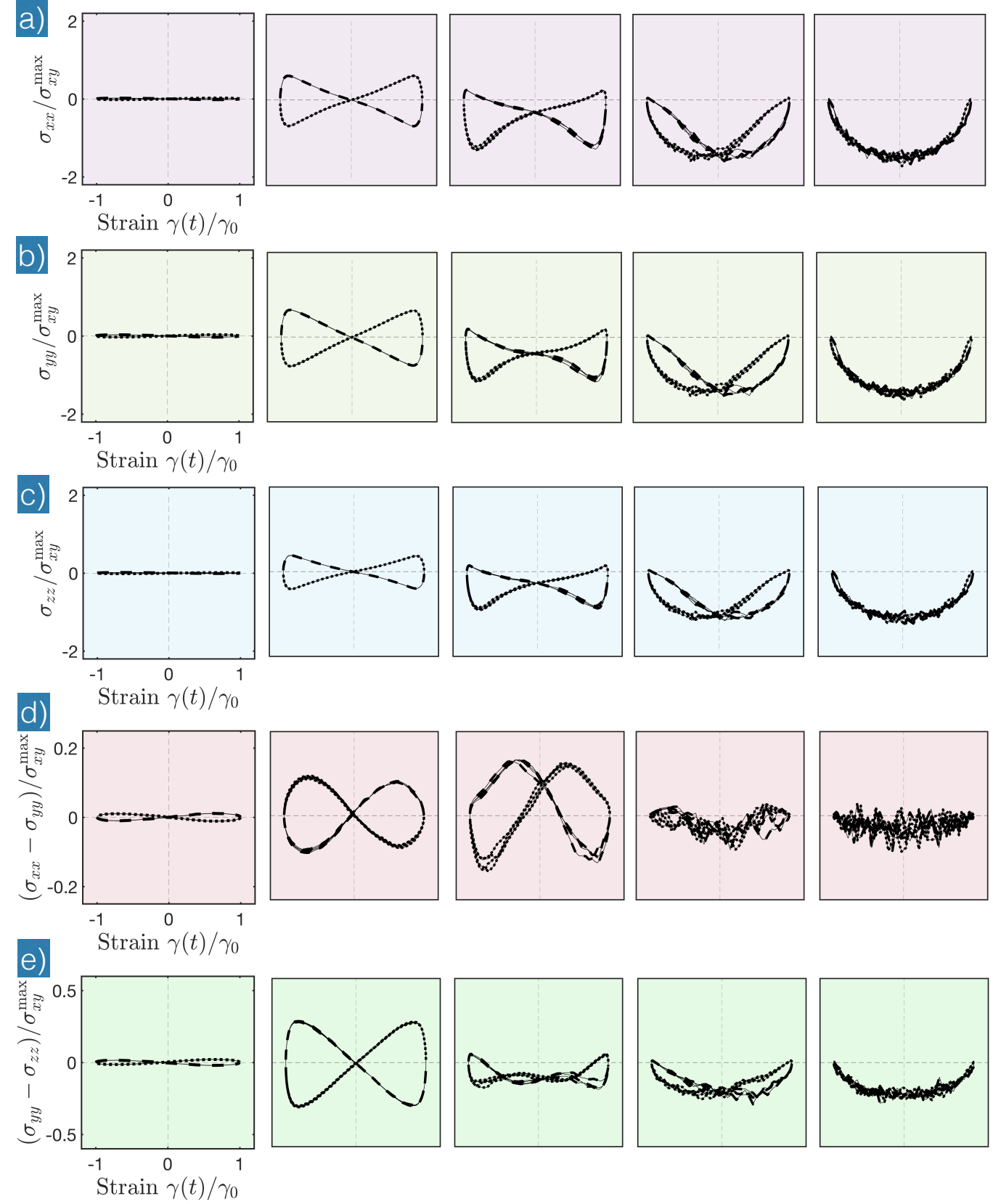

0.01

0.22

0.55

1.39

8.79

Increasing strain amplitude $\gamma_{0}$

FIG. 6. Normal stress response to oscillatory shear, predicted by simulation for $\mu=0$ and $\phi=0.55$. We present (a) $\sigma_{x x}$; (b) $\sigma_{y y}$; (c) $\sigma_{z z}$; (d) $N_{1}=\sigma_{x x}-\sigma_{y y}$ and (e) $N_{2}=\sigma_{y y}-\sigma_{z z}$. Shown are data for increasing $\gamma_{0}$, left to right, with the axis labels in the far left applicable to their whole row. To indicate the phase of each set of data, results corresponding to $\dot{\gamma}(t)>0$ are highlighted with dashes, while those for $\dot{\gamma}(t)<0$ are dotted.

\section{Quasi-Newtonian response at large amplitude}

For large strain amplitudes, the suspension microstructure becomes well established shortly after the reversing of the sign of $\dot{\gamma}(t)$, and the shear stress enters the aforementioned approximately rate-independent regime. Indeed, even the contact stress is observed to be approxi- mately rate-independent in this regime, giving a viscous contribution that leads to considerably higher viscosities in the large-amplitude compared to the small-amplitude regimes. Here, the start-up period after each reversal of flow direction, during which the microstructural quantities evolve, represents a very small portion of each oscillatory cycle. We studied the detailed evolution of the 

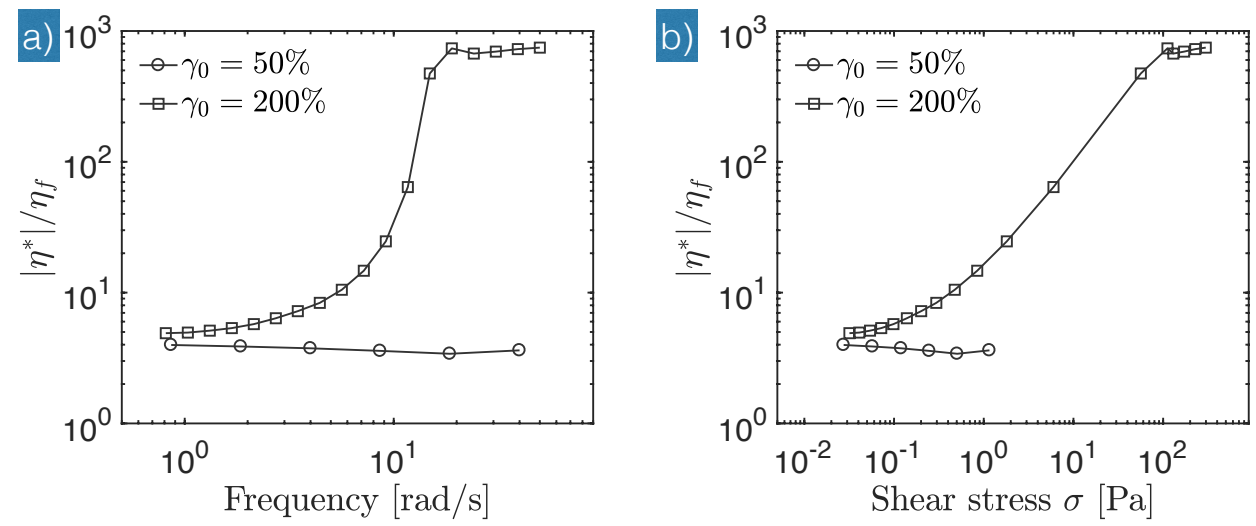

FIG. 7. Complex viscosity magnitude of cornstarch suspension as a function of strain amplitude for small and large amplitude oscillations. Shown are (a) complex viscosity magnitude against oscillatory frequency $\omega$ and (b) complex viscosity magnitude against characteristic shear stress $\sigma$.

microstructure during this phase in a separate work [11]. Indeed, the loss modulus begins to dominate again (Figure 2), with the Lissajous plot appearing to illustrate close to viscous flow. The extensive nonaffine motions during the long period of plastic deformation result in substantial irreversibility of the particle positions, Figure 5. In this case, the strain dependences of $Z_{c}$ and $A^{h}$, $A^{c}$ indicate that the suspension microstructure attains compatibility with the imposed flow shortly after changes in flow direction, and thenceforth evolve independently of $\dot{\gamma}(t)$ until $\dot{\gamma}(t)=0$, when the underlying fragility is again manifested by considerable contact breakage and subsequent reformation, which again takes place very shortly after flow start-up in the new direction. The flow in this regime, being predominantly viscous and with a considerable contact contribution after the start-up period, is consistent with the rheological law described by Boyer et al [24].

The appearance of demonstrable nonzero values of $N_{1}$ appears to be a transient effect during contact formation/breaking and microstructural evolution. For large strain magnitudes, say $\gamma_{0} \geqslant 8.79$, where the contact number and fabric become well established during the flow cycle, $N_{1}$ remains close to zero except at flow initiation. This suggests that, with respect to the flowgradient plane, the suspension is neither dilatant nor tensile. The remaining non-Newtonian character is retained, however, in $N_{2}$, which demonstrates proportionality with $\dot{\gamma}(t)$ when the microstructure is well established, illustrating a stress contribution, i.e. that of the particle contacts, that acts uniformly in $x$ and $y$, consistent with the fabric $A^{c}$ and $A^{h}$, but that is absent in $z$. Overall, the quasi-Newtonian behaviour observed here for large $\gamma_{0}$ is reminiscent of the viscous flow regime described by Boyer et al [24], in that the shear stress and normal stresses are both nonzero and rate-independent, while contacts and hydrodynamics contribute. Our results across a broad range of $\gamma_{0}$ demonstrate that this picture can be unified with that of the fragile, breakable contact networks seen for small and intermediate strains.

\section{Oscillatory rheology as a diagnostic tool for shear-induced structure}

We have identified a low strain hydrodynamicdominated viscous regime, and a high strain contactdominated viscous regime. Provided we remain in the athermal, noninertial limit, the low strain regime should remain viscous independently of the oscillatory frequency, since the dissipation is simply dominated by hydrodynamics which are governed by the rheology of the suspending liquid. We therefore expect $\left|\eta^{*}\right| \neq f(\omega)$ in this region. Conversely, any stress dependence in the shear-induced microstructure, for example frictional contacts appearing above $\sigma^{*}[6]$, should show up in a frequency sweep at the high strain limit. The simulations at $\mu=0$ and $\mu=1$, as well as the experimental data, show an increased complex viscosity magnitude $\left|\eta^{*}\right|$ and a non-negligible storage modulus at $\gamma_{0}=2$, indicating a stress contribution from shear induced structures at this point. We present experimental results for such frequency sweeps in Figure 7, for $\gamma_{0}=0.5$ and $\gamma_{0}=2$. The low strain regime shows no frequency dependence, as expected, while the large strain regime shows a sharp increase in $\left|\eta^{*}\right|$ with increasing frequency, Figure 7. In spite of the complexity of having both hydrodynamics and contacts contributing to the shear stress in differing proportions, our Lissajous plots presented above demonstrate approximately viscous rheology at large strains for $\mu=0$ and $\mu=1$. Simply considering the magnitude of the complex viscosity, therefore, we recover the stress dependence in the material viscosity. Plotting instead with the characteristic shear stress $\sigma$, we find a near linear increase in $\left|\eta^{*}\right|$ with $\sigma$, reminiscent of the steady shear rheology of a comparable suspension close to discontinuous shear thickening [6].

This result is also consistent with our simulation re- 


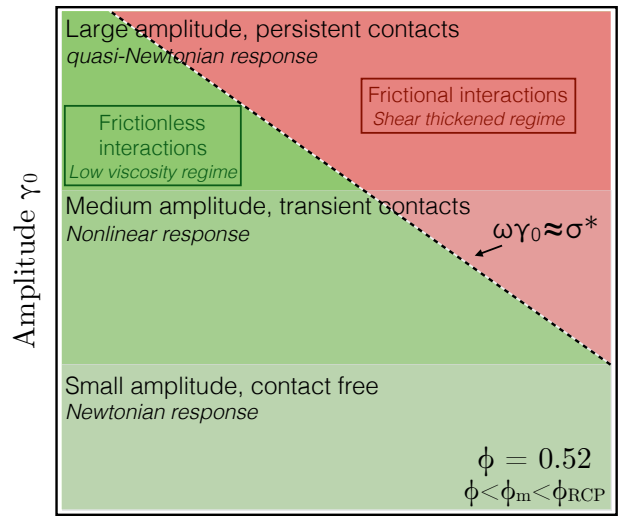

Frequency $\omega$

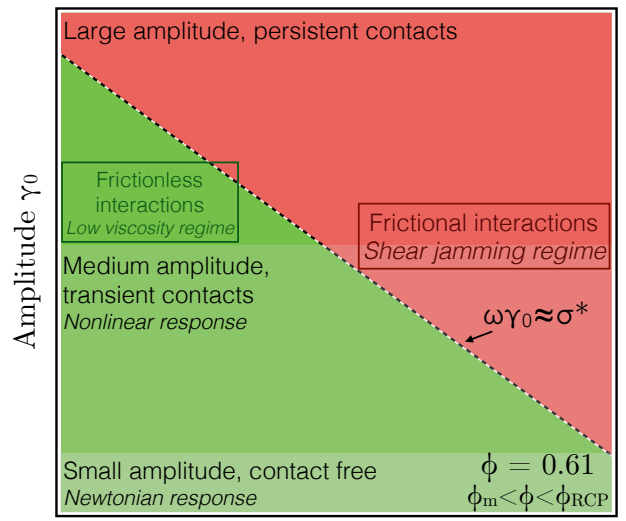

Frequency $\omega$

FIG. 8. Pipkin diagrams for the oscillatory rheology of dense suspensions at $\phi<\phi_{m}$ and $\phi_{m}<\phi<\phi_{R C P}$

sult. Comparing the $\left|\eta^{*}\right|$ response as a function of $\mu$, we find the disparity arises only at large strains. This is consistent with the notion of a stress-dependent particle friction being detected under large amplitude oscillatory flow. By this technique, therefore, we might further extend the diagnostic capabilities of non-steady flows. At small strains, a frequency sweep provides information about the at-rest configuration of the suspension, and might also be indicative of the rheology of the suspending fluid itself. At large strain amplitudes, however, we can quantify the stress dependence of any shear induced fragile structures. These might contain information about the frictional or attractive properties of particle-particle interactions, for example.

\section{CLOSING REMARKS}

The results in this work provide further testimony to the potential of non-steady rheology measurements as diagnostic tools for elucidating particle contact properties [11] and suspension stress contributors. Further work is needed to link these results to those exploring additional complexities such as particle-particle cohesive forces that may give rise to gel-like elasticity, the Brownian regime that may give rise to glassy behaviour and competition between thermal and convecting motion at low Peclet numbers, and the crossover from viscous to elastic responses at $\phi_{c}$, for example.

The nonaffine framework has been developed and successfully used to calculate the shear modulus of general disordered solids [31], including extending to specific cases of metallic glasses [52] and polymer glasses [53]. Linking the nonaffine framework to dense suspensions, and therefore to disordered systems below jamming more generally, remains a promising route, and one that can be informed by further oscillatory shear experiments and simulations.

Though a classical material for studying suspension rheology, comparing experimental cornstarch data to somewhat idealised simulation results remains tricky. In particular, the grains are spherulites with facets and edges that are difficult to define as 'hard' or 'rough', particularly when solvent effects on the surface friction are only now coming to light [54]. Overall, though, our findings demonstrate that with a sufficiently high friction coefficient, nearly-hard, bidisperse spheres can provide quantitative bulk rheology data and are useful for interpreting microsctructural evolution associated with real suspensions.

In future, oscillatory shear in suspensions might be explored as a tool for further elucidating the shear-jamming phase diagram $[55,56]$. The emerging picture of discontinuous shear thickening as a transition from lubricated to frictional particle interactions $[28,57-59]$ presents interesting consequential volume fraction effects. At low stresses, one expects the suspension viscosity to diverge at $\phi_{R C P} \approx 64 \%$, while at high stresses the divergence occurs at $\phi_{m} \approx 58 \%$. In the present article we remain below both critical values of $\phi$ (accounting for the shape effects in our cornstarch suspension), such that we stay away from jamming or shear-jamming at all times. By increasing the volume fraction to $\phi_{R C P}<\phi<\phi_{m}$, though, we might explore the regime where shear-jamming occurs above some critical strain, which could be explored as an extreme strain-hardening effect [17]. Such shear-jamming might be aided by the inherent capability of dense packings to exploit pre-existing, potential load-bearing structures at small strains [60]. To this end, we construct tentative Pipkin diagrams at two representative volume fractions, to illustrate the general rheological regimes expected tying in the stran-amplitude dependence explored in this work, Figure 8. Here, we indicate a line of fixed stress $\sigma^{*}$ at which particle-particle interactions become frictional. For simplicity we show this as a sharp transition, but in practice the onset of friction occurs over a range of stress [61]. At low volume fractions this transition might be observed as a shear thickening transition, 
similar to that presented in this work, while at higher volume fractions it is anticipated that the shear jamming regime is entered.

Our findings demonstrate the unique flow map of nearly hard particle suspensions under oscillatory shear. Keeping the strain small allows us to probe the rheology of the interstitial fluid; large strains allow us to build up a shear induced microstructure and quantify its contribution to the viscosity. The character of the intermediate regime can reveal the susceptibility of the load-bearing contact network to incompatible flows. More generally, our results may lay a template for future use of oscillatory flows as diagnostic tools for characterising challenging in- dustrial suspensions $[62,63]$, and may also inform future microstructurally-based constitutive relations for dense flowing systems [64].

\section{ACKNOWLEDGEMENTS}

Helpful discussions with Jin Sun, Meera Ramaswarmy, Michiel Hermes and Rangarajan Radhakrishnan are gratefully acknowledged. $\mathrm{CN}$ is supported by the Maudslay-Butler Research Fellowship at Pembroke College, Cambridge; ZX is supported by the National University of Defense Technology, China.
[1] É. Guazzelli and J. F. Morris, A Physical Introduction to Suspension Dynamics, Cambridge Texts in Applied Mathematics (Cambridge University Press, 2011).

[2] A. J. Liu and S. R. Nagel, Nature 396, 21 (1998).

[3] A. Ikeda, L. Berthier, and P. Sollich, Physical Review Letters 109 (2012).

[4] E. Lerner, G. Düring, and M. Wyart, Proceedings of the National Academy of Sciences of the United States of America 109, 4798 (2012).

[5] Y. Gu, S. Chialvo, and S. Sundaresan, Physical Review E 90, 1 (2014).

[6] B. E. Guy, M. Hermes, and W. Poon, Physical Review Letters 115 (2015).

[7] C. Ness and J. Sun, Physical Review E 91, 012201 (2015).

[8] S. Chialvo, J. Sun, and S. Sundaresan, Physical Review E 85 (2012).

[9] M. E. Cates, J. Wittmer, J.-P. P. Bouchaud, and P. Claudin, Physical Review Letters 81, 1841 (1998).

[10] F. Gadala-Maria and A. Acrivos, Journal of Rheology 24, 799 (1980).

[11] C. Ness and J. Sun, Physical Review E 93, 012604 (2016).

[12] N. Y. Lin, C. Ness, M. E. Cates, J. Sun, and I. Cohen, Proceedings of the National Academy of Sciences, 201608348 (2016).

[13] J. Benbow and J. Bridgewater, Paste flow and extrusion (Oxford University Press, 1993).

[14] J. M. Brader, M. Siebenbürger, M. Ballauff, K. Reinheimer, M. Wilhelm, S. J. Frey, F. Weysser, and M. Fuchs, Physical Review E 82 (2010).

[15] D. Fiocco, G. Foffi, and S. Sastry, Physical Review E 88 (2013).

[16] P. Sollich, F. Lequeux, P. Hébraud, and M. E. Cates, Physical Review Letters 78, 2020 (1997).

[17] S. Khandavalli and J. P. Rothstein, Rheologica Acta 54, 601 (2015).

[18] F. Zimber, J. E. Kollmer, and T. Peschel, Physical Review Letters 111 (2013).

[19] R. Pastore, M. Pica Ciamarra, and A. Coniglio, Granular Matter 14, 253 (2012).

[20] N. Y. Lin, B. M. Guy, M. Hermes, C. Ness, J. Sun, W. C. Poon, and I. Cohen, Physical Review Letters 115, 228304 (2015).

[21] E. J. Hinch, J. Fluid Mech. 686, 1 (2011).

[22] J. Mewis and N. J. Wagner, Journal of Non-Newtonian Fluid Mechanics 157, 147 (2009).
[23] M. E. Cates and M. Wyart, Rheologica Acta 53, 755 (2014).

[24] F. Boyer, É. Guazzelli, and O. Pouliquen, Physical Review Letters 107 (2011).

[25] GDR Midi, European Physical Journal E 14, 314 (2004).

[26] P. Jop, Y. Forterre, and O. Pouliquen, Nature 441, 727 (2006).

[27] J. R. Royer, D. L. Blair, and S. D. Hudson, Physical Review Letters 116 (2016).

[28] R. Seto, R. Mari, J. F. Morris, and M. M. Denn, Physical review letters 111, 218301 (2013).

[29] J. G. Nam, K. H. Ahn, S. J. Lee, and K. Hyun, Rheologica Acta 50, 925 (2011).

[30] S. A. Rogers, B. M. Erwin, D. Vlassopoulos, and M. Cloitre, Journal of Rheology 55, 435 (2011).

[31] C. E. Maloney and A. Lemaitre, Physical Review E 74 (2006).

[32] J. F. Morris and J. F. Brady, Journal Of Fluid Mechanics 312, 223 (1996).

[33] A. Sierou and J. F. Brady, Journal of Fluid Mechanics 506, 285 (2004).

[34] D. J. Pine, J. P. Gollub, J. F. Brady, and a. M. Leshansky, Nature 438, 997 (2005).

[35] M. Souzy, P. Pham, and B. Metzger, Physical Review Fluids 042001(R), 1 (2016).

[36] K. Hyun, M. Wilhelm, C. O. Klein, K. S. Cho, J. G. Nam, K. H. Ahn, S. J. Lee, R. H. Ewoldt, and G. H. McKinley, Progress in Polymer Science 36, 1697 (2011).

[37] M. Wilhelm, Macromolecular Materials and Engineering 287, 83 (2002).

[38] C. O. Klein, H. W. Spiess, A. Calin, C. Balan, and M. Wilhelm, Macromolecules 40, 4250 (2007).

[39] K. S. Cho, K. Hyun, K. H. Ahn, and S. J. Lee, Journal of Rheology 49, 747 (2005).

[40] R. H. Ewoldt, A. E. Hosoi, and G. H. McKinley, Journal of Rheology 52, 1427 (2008).

[41] S. A. Rogers, Journal of Rheology 56, 1129 (2012).

[42] A. J. Giacomin, R. B. Bird, L. M. Johnson, and A. W. Mix, Journal of Non-Newtonian Fluid Mechanics 166, 1081 (2011).

[43] F. Peters, G. Ghigliotti, S. Gallier, F. Blanc, E. Lemaire, L. Lobry, and L. E. G, Journal of Rheology 60, 715 (2016).

[44] S. Plimpton, Journal of Computational Physics 117, 1 (1995). 
[45] P. A. Cundall and O. D. L. Strack, Géotechnique 29, 47 (1979).

[46] Full model details and justification provided in Refs [6567]; steady state rheological phase space predicted by our model is given in Ref [7]; the suitability of our model for capturing the rheology of typical model suspensions such as $2 \mu \mathrm{m}$ charged-stabilised silica in glycerol is demonstrated in Ref [20].

[47] J. R. Royer and P. M. Chaikin, Proceedings of the National Academy of Sciences 112, 49 (2015).

[48] A. Fall, F. Bertrand, G. Ovarlez, and D. Bonn, Journal of Rheology 56, 575 (2012).

[49] M. Hermes, B. M. Guy, G. Poy, M. E. Cates, M. Wyart, and W. C. K. Poon, Journal of Rheology 60, 905 (2016).

[50] Following Ref [68], we verified that the strain control obtained with our stress-controlled rheometer is such that measured nonlinear responses obtained are dominated by material nonlinearities. The inertia $I$ of the measuring device is of order $10^{-6} \mathrm{kgm}^{2}$ [69]. The geometry factor of a cone-and-plate rheometer of angle $\epsilon$ and radius $R, k=3 \epsilon / 2 \pi R^{3} \approx 10^{2}$. For $\omega=50 \mathrm{rad} / \mathrm{s}$, therefore, we require $G \gtrsim 0.25 \mathrm{~Pa}$ (following Ref [70]), to rule out instrument inertia, suggesting that instrument inertia plays only a small role. Moreover, we re-measured the low strain amplitude data at a frequency of $0.5 \mathrm{rad} / \mathrm{s}$ and found quantitatively comparable rheology, further suggesting that our results are not heavily influenced by instrument inertia.

[51] E. DeGiuli, G. Düring, E. Lerner, and M. Wyart, Physical Review E 062206, 1 (2014).

[52] A. Zaccone, P. Schall, and E. M. Terentjev, Physical Review B 90 (2014).

[53] V. Palyulin, C. Ness, R. Milkus, R. Elder, T. Sirk and A. Zaccone, in preparation.

[54] L. O. Galvez, S. de Beer, D. van der Meer, and A. Pons, (2017), arXiv:1703.05601.

[55] D. Bi, J. Zhang, B. Chakraborty, and R. P. Behringer, Nature 480, 355 (2011).

[56] M. P. Ciamarra, R. Pastore, M. Nicodemi, and A. Coniglio, Physical Review E 84 (2011).

[57] N. Fernandez, R. Mani, D. Rinaldi, D. Kadau, M. Mosquet, H. Lombois-Burger, J. Cayer-Barrioz, H. J. Herrmann, N. D. Spencer, and L. Isa, Physical Review Letters 111 (2013).

[58] C. Heussinger, Physical Review E 88 (2013).

[59] M. Wyart and M. E. Cates, Physical Review Letters 112 (2014).

[60] M. C. Jenkins, M. D. Haw, G. C. Barker, W. C. K. Poon, and S. U. Egelhaaf, Physical Review Letters 107 (2011).

[61] R. Mari, R. Seto, J. F. Morris, and M. M. Denn, Journal of Rheology 58, 32 (2014).

[62] R. Gallego, J. Arteaga, C. Valencia, and J. Franco, Chemical Engineering Science 134, 260 (2015).

[63] C. Durán, Y. Fayolle, Y. Pechaud, A. Cockx, and S. Gillot, Chemical Engineering Science 141, 154 (2016).

[64] J. Sun and S. Sundaresan, Journal of Fluid Mechanics 682, 590 (2011).

[65] R. C. Ball and J. R. Melrose, Physica A: Statistical Mechanics and its Applications 247, 444 (1997).

[66] S. Kim and S. J. Karilla, Book (Butterworth-Heinemann, 1991) p. 503.

[67] C. Ness and J. Sun, Soft Matter 12, 914 (2016).

[68] D. Merger and M. Wilhelm, Rheologica Acta 53, 621 (2014).
[69] I. M. Krieger, Journal of Rheology 34(4), 471 (1990).

[70] R. H. Ewoldt, M. T. Johnston, and L. M. Caretta, Complex Fluids in Biological Systems 7, 3866 (2015). 